\title{
Near-Infrared Radiaton: An All Pervading Yet Lesser Known Environmental Stressor
}

\author{
Hyacinth Highland, Linz-Buoy George \\ Department of Zoology, Biomedical Technology and Human Genetics, Gujarat University, \\ Ahmedabad, Gujarat. India.
}

*Corresponding Author: Hyacinth Highland, Department of Zoology, Biomedical Technology and Human Genetics, Gujarat University, Ahmedabad, Gujarat. India.

\begin{abstract}
Current research has focused on evaluating the possible impact of environmental agents on the sperm cell, since it functions to carry the male genome to the next generation. Among the myriads of environmental factors known to afflict human life, near infrared radiation (840 -1000 nm), warrants increased research attention, due to its escalating use in varied fields; however research in this area is wanting. Researchers have demonstrated that the oxidative damage induced in several tissues by near infrared radiation is through the generation of free radicals and reactive oxygen species (ROS) and that mitochondrial cytochromes, porphyrins and flavoproteins possess absorption bands in the NIR and hence these wavelengths could manifest potential biological effects. While most research has been centered on the skin and eye, there is a paucity of data on the impact of NIR on reproductive tissues, specifically on spermatozoa.

Technological advances have led to the development of NIR-lasers; but there are conflicting reports regarding their effect and the exact nature of their influence remains an enigma. Several reports suggested a positive, short-term effect on the sperm motility and membrane function with no significant DNA damage. Investigations carried out earlier at our laboratory, clearly indicated that whole body and abscopal exposure of adult, male albino rats to moderate duration near-IR radiation proved deleterious to reproductive tissues. Conversely, our in-vitro studies confirmed that short duration, low-level $900 \mathrm{~nm}$ nearinfrared radiation exposure manifested hormetic effects on the sperm cell, triggering sustained sperm motility. Thus, the intensity and duration of exposure in the near-infrared region have a significant bearing on the cell response. Spermatozoa are most vulnerable to exogenous attack and may be severely afflicted by the all pervading near-infrared sources. Hence this study holds special significance.
\end{abstract}

Keywords: Near Infrared radiation, human spermatozoa, motility activation

\section{INTRODUCTION}

It seems unwarranted to reiterate that in the past several decades, human life on this planet has faced assault from innumerable deleterious environmental agents, the barrage of which has left mankind living on the edge. A multitude of environmental toxicants and agents have been identified, researched and even proven to have destroyed a large number of molecular pathways and functions in every known mammalian cell. Environmental degradation has been intrinsically implicated with a direct impact on the sperm cell, leading to a loss of sperm fertilizing ability and possibly accounting for the reported decline in human fertility ( Carlsen et al., 1992; Roser, 2016).

Across the globe, fundamental research has accordingly been directed towards understanding the possible impact on the sperm cell, since this cell works as a conduit, carrying the human genome across generations. In addition, as recognized earlier on (Overstreet et al., 1988) the human sperm is most vulnerable to exogenous attack from environmental sources. Although the alleviation of infertility is a primary concern for the human research force, what really sparks Scientist's attention, is the possibility of deleterious damage to the DNA and the prospect of its transmission to the ensuing generation.

What appears to be most intriguing is that this cell is naturally a protected cell, fortified through a barricade of protection mechanisms, including the blood-testis-barrier (BTB), one of the tightest blood-tissue barriers which shields the highly specialized and metabolically inactive developing spermatozoa (Cheng and Murg, 2012) against environmental toxicants, xenobiotics and estrogenic mimics. 
Additionally, the seminal plasma is a rich source of protective ammunition, guarding the sperm from a countless xeno-toxicants and microorganisms. Despite the innate shielding mechanisms, spermatozoa have not escaped the torrential assault from electromagnetic radiation that represents a persistently hazardous component of the environment. Specifically, the sperm cell has been afflicted by increased radiation exposure since this cell is at risk both in the macro (workplace) and microenvironments. It is therefore imperative that systematic investigation is carried out to evaluate the molecular changes and functional insult that occurs in spermatozoa on exposure to certain commonly used forms of radiation.

With the advent of high-end electronic technology, one form of electromagnetic radiation viz., Infrared radiation has been exploited extensively, with widespread applications in every locale of human life. Conversely, very little attention has been paid to analysing the effect of infrared radiation on the human sperm. Moreover, infrared radiation occurs in a spectrum of wavelength from 840 to $10^{6} \mathrm{~nm}$, comprising four spectral regions, with a concomitant decrease in energy. of these, the near infrared radiation (840-1000 $\mathrm{nm})$ is used rampantly for varied applications while lower energy far infrared in used in medical fields. Hence, the goal of this review was to put into perspective the manifold effects due to occupational and incidental exposure to lowlevel near Infrared radiation, with special emphasis on human spermatozoa.

A unique characteristic of Infrared radiation (IR-R) is its long wavelength, coupled with low energy and hence IR-R was earlier regarded as harmless and as a safe heat therapy or hyperthermia. However, IR-R is known to penetrate the skin up to 3-4 $\mathrm{mm}$ (Lenz, 1995), warming tissues and stimulating blood circulation to enhance the supply of nutrients and oxygen to the cells as well as, activate proteins such as collagen, elastin and release nitric oxide. This long wavelength form of radiation, termed far-infrared, helps to improve the circulation of blood, which draws in growth factors that in turn, expedites the recovery of the injured muscle or tissue and accelerates the release of dead cells. Hence, IR-R has been used to treat skin problems, dandruff, acne, blackheads, psoriasis, eczema, smoothing of wrinkles, joint diseases, skin injuries, etc. As the source for infrared saunas, it works in the treatment of chronic health problems, such as high blood pressure, congestive heart failure and rheumatoid arthritis, to improve and stabilize blood circulation, as a relaxant of muscles, bronchitis, asthmatic disorders; IR-R helps in immunity and to alleviate fatigue and exhaustion of the human body; it is useful in inflammation and diminishes pain in arthritic and rheumatic diseases and herniated discs; It has also been implicated in aiding kidney function and in procedures for the reduction of cellulite and fat. Certain medical applications do employ near-infrared radiation also.

In the late seventies, Zeret et al. (1976) inferred that Infrared radiation is one of the many contributory factors resulting in molecular damage in cells and tissues. It was also postulated that the high water content in living cells and tissues works as the principal absorber of infrared radiation, which in turn manifests effects on cellular bio-molecules. Years later, Cho et al. (2009) confirmed that the oxidative damage induced by infrared radiation is through the generation of free radicals and reactive oxygen species (ROS). Measuring the hydroxyl free radicals generated in sperm cells, as a function of wavelength, it was observed that short wavelength radiation (Lubart et al., 1997) was more effective in stimulation of hydroxyl radicals. Further, Ban Frangez et al. (2015) have pointed out that intensity and duration of irradiation are of prime importance in ascertaining its effects.

\section{UBIQUITOUS: NEAR INFRARED RADIATION}

As mentioned, near infrared radiation of wavelength range 840 to $1000 \mathrm{~nm}$ has been extensively used in varied fields, in the past few decades. It has been well documented that the natural source of infrared radiation is the sun and that the irradiation at the earth's surface is $0.9 \mathrm{w} / \mathrm{cm} 2$ (Hudson, 1969). However, we are now increasingly being exposed to infrared radiation, both occupationally and environmentally, due to its wide-ranging use in diverse technology. Infrared light is used in industrial, scientific and medical applications. Infrared heating can be used in industrial manufacturing processes, curing of coatings, forming of plastics, annealing, plastic welding and print drying. Infrared heaters have replaced convection ovens and contact heaters and are commonly used in infrared modules, classified by the wavelength they emit: Near infrared (NIR) and Far infrared emitters (FIR). 
Near Infrared radiation (NIR-R) has the shortest wavelength and highest energy among the forms of Infrared radiation. It is an intrinsic part of communications signals, fiber optic telecommunication, remote controls and optical fibre communications; for information transmission using analogue, digital signals or data links over short distances such as that between computers or mobile phones. Infrared sensors can detect heat from the body and are also effectively used for security lights and burglar alarms. Military applications include target acquisition, surveillance and night vision, homing and tracking. Non-military uses include thermal efficiency analysis, environmental monitoring, industrial facility inspections, remote temperature sensing, short-ranged wireless communication; most remote controls for TV, DVD players, projectors, operate by on pulses of infrared, etc. Can it be argued that there is no escape from the emissions of near infrared radiation?

\section{Adverse Effects - Caveats from Literature}

The primary harmful effect of near infrared radiation (IR) is thermal injury, along with scaling and hyper-pigmentation of skin and pain. The main biological effects of near-IR radiation are infrared cataracts (Linz-Buoy, 1990; Aly and Mohamed, 2011) and flash drying of the cornea. Near infrared radiation wavelengths are close to the visible light wavelengths and are transmitted to a small extent to the retina; permanent retinal damage can occur if exposure is prolonged. As wavelengths increase beyond $1000 \mathrm{~nm}$ levels, the radiation is no longer transmitted to the retina but corneal flash burn injuries can still be caused. Using spin trapping technology, König et al. (1996) have shown that short-wavelength NIR traps $(<800 \mathrm{~nm})$ induce UVA-like biological effects inclusive of oxidative stress. According to a publication by Ban Frangez et al. (2015) mitochondrial cytochromes, porphyrins and flavoproteins possess absorption bands in the NIR and hence these wavelengths manifest potential biological effects.

Robert et al. (2015) have aptly remarked that the human skin is daily exposed to sun rays, which include not only ultraviolet radiation, but also a substantial quantity of infrared (IR) radiation. In-vitro research on normal human fibroblasts, by these scientists have revealed that even under low irradiance with single or very few repeated doses, near-infrared irradiation produces free radicals, triggers major changes in the expression of the type I collagen and elastin network, impairs the dermal-epidermal junction, upregulates several matrix metalloproteinases and has an impact on the expression of key genes of the extracellular matrix. They thus conclude that chronic or discretionary exposure to near-IR could play a role that is more important than expected in premature skin aging.

Despite the evidence, this radiation was regarded as comparatively safe and hence there was a paucity of research in this direction. In the recent past the effect of infrared radiation on the eye and skin received some research attention, but effects of NIR on the sperm or reproductive system remained on the back burner. Tanaka et al. (2011) previously reported that near-infrared irradiation (NIR) can penetrate the skin and non-thermally affects dermis and superficial muscles. The nonthermal effects of simulated solar radiation or near-infrared was then recognized and further reported to increase subcutaneous and bone marrow adipocytes, CD34 - positive hematopoietic stem cells and cortical bone mass. It also induced putative, non-thermal damage of bone marrow, which was shown to be mediated by apoptosis. These reports made it clear that infrared radiation could affect internal organs and hence the reproductive tissues were also at risk.

Tanaka et al. (2012) further demonstrated that the cell viability of cultured lung adenocarcinoma cells was significantly decreased after near-infrared irradiation at 20 $\mathrm{J} / \mathrm{cm}$, with increase in Apoptotic cells. In addition these researchers have shown that nearinfrared and doxorubicin inhibited the tumor growth of melanoma cells suggesting that nearinfrared irradiation can non-thermally induce cytocidal effects in cancer cells as a result of activation of the DNA damage response pathway. Since NIR has immense usage, several authors including Vatansever and Hamblin (2012) have described its varied biological effects, but yet little concern was raised with regard to the impact of near infrared radiation on reproductive tissues.

\section{Beneficial Effects of Nir - The Paradigm Shift}

In successive years, NIR effects were scrutinized from an entirely different angle and the focus turned towards the curative efficacy of near-infrared radiation, with emphasis on its usage in lasers. The beneficial effects in tissue injury due to treatment with light in the near- 
infrared region of the spectrum were demonstrated by Tang et al. (2013). It was also proven that short wavelength near-infrared laser can stimulate the functional hair cells (Xia et al., 2014).

It was aptly established by $\mathrm{Li}$ et al. (2014) that a certain photothermal effect (PE) plays a major role in the near-infrared laser interaction with biological tissue and further these authors have confirmed that the alteration of sodium current kinetics on neuron cells in-vitro was due to a modulation effect at 980-nm infrared exposure. It was then stated by Giacci et al. (2015) that near-infrared light therapy (NIR-LT) improves functional and morphological outcomes in a range of central nervous system injuries in vivo, possibly by reducing oxidative stress. This observation threw new light on the hitherto hypothesis that infrared radiation manifests adverse effects through the generation of free radicals. Reports that NIR effects vary depending on wavelength or intensity of irradiation offered a sound explanation for the modulatory effects of NIR. Subsequently a combinational therapy employing NIR, photobiomodulation (PBM) evolved. Therefore it was inferred that low level exposure to NIR at short duration, results in a hormetic effect while longer duration, shorter wavelengths could trigger free radicals and ROS which in turn could have serious repercussions.

In an in-depth study Eells et al. (2015) provided evidence that 'photobiomodulation' (PBM) can restore the function of damaged mitochondria, upregulate the production of cytoprotective factors and prevent apoptotic cell death and hence this NIR component works efficiently in the treatment of soft tissue injuries and acceleration of wound healing. The therapeutic effects of PBM have been hypothesized to result from intracellular signaling pathways triggered when NIR photons are absorbed by the mitochondrial photoacceptor molecule, cytochrome c oxidase, culminating in improved mitochondrial energy metabolism, increased cytoprotective factor production, promotes cell survival and exerts anti-inflammatory actions.

Further, it was shown that PBM did not alter cytochrome oxidase activity in the retina or in cultured cells and this therapy was found to inhibit superoxide production, simultaneously preserving Mn-SOD expression. The use of NIR through photobiomodulation was then demonstrated to ameliorate lesions of diabetic retinopathy, reduce oxidative stress and cell death in-vitro. These biomodulatory manifestations of the near-infrared region are in stark contrast to earlier reports indicating a cell apoptotic effect through production of free radicals. Hence, newer strategies employed in the use and emission of NIR, yield biostimulatory and biomodulatory effects which far outweigh the deleterious effects of Near Infrared Radiation.

\section{Dual Effects of UV and NIR Radiation}

Evaluation of the dual effects of ultraviolet and infrared radiation, further paved the way for understanding the biological effects of near infrared radiation viewed from a new dimension.

In a most comprehensive review, Horlicks (2016) has put into perspective the many health benefits that have been associated with exposure to sunlight, ultraviolet A (UVA) ultraviolet B (UVB), visible and infrared radiation. Gonzalez et al. (2015) have stated that infrared radiation exposure after ultraviolet B irradiation increases skin damage without protecting the tissue, while infrared radiation exposure before ultraviolet B irradiation showed a protective effect against ultraviolet skin damage.

\section{Is Near Infirared Detrimental To The Sperm Cell?}

The main purpose of this review is to shed light on the effect of near infrared radiation on the sperm cell. Recently, Sakkas and Alvarez (2010) have suggested that induced DNA aberrations could result in a protamination deficiency that would subsequently render the sperm DNA increasingly vulnerable to a variety of environmental stressors. Does near-infrared radiation function as a genomic stress inducer?

Firestone et al. (2012) have monitored the effects of low-level laser light exposure on the motility of spermatozoa and on DNA damage. In their experiment, a significant increase in motility was observed when samples were exposed to a 30-second infrared pulse of 905 $\mathrm{nm}$, a wavelength known to increase the activity of the light-sensitive Cytochrome $\mathrm{C}$ oxidase in the mitochondrial electron transport chain. This increase in motility was found to be most prominent 30 minutes after the treatment in oligospermic and asthenospermic samples $(85 \%$ increase; $\mathrm{P}<0.001)$ and specifically significant 
changes in sperm motion kinetics were observed.

A plausible explanation for this phenomenon of motility activation was that low-level NIR irradiation could stimulate the production of adenosine triphosphate and thereby increase energy supply to the sperm cell. In a separate study, Yazdi et al. (2013) have described the effects of similar irradiation on human sperm motility. Earlier, Lubart et al. (1997) have reported that $780 \mathrm{~nm}$ irradiation could bring about changes in calcium transport in mammalian sperm mitochondria and plasma membranes. This observation was experimentally established by Friedmann et al. (1997) that $780 \mathrm{~nm}$ Infrared inhibits $\mathrm{Ca} 2+$ uptake by sperm mitochondria and enhances $\mathrm{Ca} 2+$ binding to sperm plasma membranes.

Furthermore it was emphasized by Firestone et al. (2012) that there was no significant increase in DNA damage as compared with control samples. This observation was a turning point in the understanding of the effects of low-level NIR, indicating that such exposure appears to have a positive short-term effect on the motility and membrane function of treated spermatozoa but did not cause any increase in DNA damage even when measured up to 2 hours.

We must recall here that Cho et al. (2009) have proven and emphatically stated that near infrared radiation causes oxidative DNA damage induced by generation of ROS. This is due higher intensities and longer durations of exposure of the near IR wavelength range. According to Zan-Bar et al. (2005), the effects of such irradiation are mediated through reactive oxygen species (ROS). Moreover, high ROS level can lead to cell death (by ATP depletion and lipid peroxidation), at low level, ROS can play a major role in activation of many cellular processes. In the case of spermatozoa, the accepted paradigm states that ROS, including superoxide anion, $\mathrm{H}_{2} \mathrm{O}_{2}$, and reactive nitrogen species such as nitric oxide (NO) can cause sperm hypercapacitation and the acrosome reaction. The energy generation involved is, in fact, an essential parameter in NIR irradiation and hence the duration for the cell to obtain metabolites from the medium is a key link to evaluating the final effects of the incident radiation. In lieu of these rather confusing and paradoxical reports, further research is imperative to delve into the exact nature and effects of short duration near infrared radiation.

\section{EXPERIMENTAL OBSERVATIONS}

A large quantum of research energy has been spent in trying to unearth the 'NIR-effect' conundrum. There is little consensus on whether the low wavelength range NIR has detrimental or beneficial effects on sperm function. With the main aim of investigating the consequence of the near-infrared radiation exposure, an in-depth study was carried out earlier at our laboratory designed to investigate the consequent metabolomic and functional alterations in male reproductive tissues. Healthy adult male albino mice were exposed to near infrared radiation (IR-emission wavelength $900 \mathrm{~nm}$ ), for the periods of $3,7,15$ and 30 days, for varying durations of $10,15,20,30$ and 45 minutes each day, to determine the possible influence on the reproductive function. Age matched, unexposed, normal animals were selected by randomization as controls. The Infrared exposure source, duration and distance were formulated based on sources used in biomedical applications.

The study specifically indicated that infrared irradiation impaired testicular steroidogenesis where the cholesterol levels were lowered, while activities of 3- $\beta$ and 17- $\beta$ hydroxysteroid dehydrogenase were elevated, correlated with a decline in the serum testosterone levels (Fig.1). Infrared radiation also brought about significant reduction in oxidative metabolic enzymes, metabolites and total protein content of reproductive tissues. In correlation to the metabolic alterations, antioxidant enzymes viz., Superoxide dismutase, Catalase, Glutathione peroxidase and reductase were significantly lowered with concomitant elevation of lipid peroxidation. In addition, microscopic observation indicated that infrared radiation damaged the histoarchitecture of all target reproductive tissues studied (Highland et al., 2003). In particular, infrared radiation exposure had an evidently detrimental effect on the testis. Tissue atresia was markedly visible with increased vacuolization, spermatogenic arrest and germ cell denudation in the testis of the exposed groups of animals. The cauda epididymis appeared to suffer pronounced injury on irradiation, with a significant decline in sperm count, motility, viability and concomitant loss of sperm membrane and nuclear integrity. 


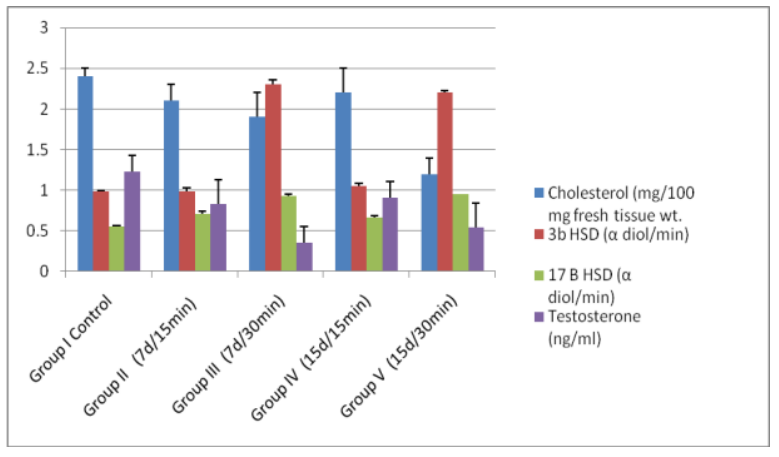

Fig1. Effect of whole body Infrared exposure on mouse testicular steroidogenesis

These early investigations carried out in our laboratory therefore, clearly indicated that whole body and abscopal exposure of adult, male albino rats to a moderate dose of radiation proved deleterious to male reproductive tissues (Highland et al., 2003; Highland et al., 2005). Research also reflected that the fertility rate of the IR treated animals was lowered. ANOVA data indicated that the altered effects were highly significant in mice exposed to IR over longer periods and durations. One main outcome of the study was the indication that there was a marked dose-duration dependent effect on the reproductive tissues.

It must be mentioned here that the animals were kept in air-conditioned environments and the temperature recorded within the exposure chamber before and after treatment suggested no significant elevation of temperature within the chamber. Hence, it is possible that infrared radiation manifests its effects through molecular excitation and photo-oxidation phenomena, unrelated to tissue heating. These findings could be correlated with the non-thermal effects of near infrared radiation described by Tanaka et al. (2011) on other tissues. Konig et al. (1996) have demonstrated that near infrared radiation can stimulate multiphoton processes in single living cells resulting in membrane permeability changes and oxidative stress. In confirmation, Cho et al. (2009) have also endorsed the fact that infrared radiation causes oxidative DNA damage triggered by generation of ROS. As an outcome of their research, Mitsunaga et al. (2012) have described the development of nearinfrared (wavelength range 840-1000 $\mathrm{nm}$ ) photoimmunotherapy to target cancer cells and reduce their survival, confirming the potential of these rays.

The deleterious effects on exposure to near-IR was further emphasized more recently when research by Robert et al. (2015) indicated that NIR irradiation (NIR-900nm) produces free radicals, triggers major changes in the expression of the type I collagen and elastin network, impairs the dermal-epidermal junction, upregulates several matrix metalloproteinases and has an impact on the expression of key genes.

\section{In-Vitro Investigations}

While the data accrued at our laboratory earlier accentuates the detrimental impact of Infrared radiation, our in-vitro investigations that followed proved otherwise. At lower doses, (shorter duration, lower lux intensity), we observed that infrared radiation proves to be stimulatory and beneficial, activating key enzymes and gene expression. In an in-vitro study, media washed human spermatozoa were maintained under sterile conditions, and exposed to a near-IR source for brief durations of 30,60, 90, 120 and 150 seconds followed by evaluation of motility, viability, biochemical indices and DNA fragmentation. The study revealed that in the time frame between 60 and 120 seconds IR exposure, the sperm cells manifested sustained motility (Table 1), enhanced enzyme activity, increased uptake of Calcium, with no significant alteration in the nuclear DNA integrity. These observations are under scrutiny and to be published shortly.

Table1. Percent sperm motility in Control and 900 nm NIR-irradiated human spermatozoa (in-vitro data)

\begin{tabular}{|c|c|c|c|c|c|}
\hline \multirow{2}{*}{$\begin{array}{l}\text { Time } \\
\text { (Sec) }\end{array}$} & \multirow{2}{*}{$\begin{array}{l}\text { Control } \\
(\% \\
\text { motility })\end{array}$} & \multicolumn{4}{|c|}{ IR Irradiated (\% motility) } \\
\hline & & I & II & III & IV \\
\hline 0 & $87 \pm 11$ & $85 \pm 03$ & $87 \pm 14$ & $85 \pm 12$ & $85 \pm 16$ \\
\hline 20 & $72 \pm 09$ & $74 \pm 11$ & $70 \pm 09$ & $72 \pm 09$ & $79 \pm 07$ \\
\hline 30 & $70 \pm 05$ & $68 \pm 07$ & $68 \pm 03$ & $69 \pm 05$ & $73 \pm 08$ \\
\hline 60 & $52 \pm 11$ & $67 \pm 10$ & $66 \pm 11$ & $69 \pm 08$ & $60 \pm 11$ \\
\hline 90 & $38 \pm 07$ & $68 \pm 06$ & $66 \pm 10$ & $66 \pm 12$ & $60 \pm 05$ \\
\hline 120 & $35 \pm 08$ & $56 \pm 11$ & $60 \pm 05$ & $65 \pm 05$ & $57 \pm 07$ \\
\hline 150 & $23 \pm 08$ & $55 \pm 08$ & $52 \pm 09$ & $55 \pm 07$ & $50 \pm 12$ \\
\hline
\end{tabular}

Values are Mean \pm S.E. Experiment Nos. I.II.III.IV. $n=24$ in each experiment.

Our findings are supported by evidence from current literature which suggest that Infrared energy proves hormetic provided the dose and duration are kept at extremely low levels. In corroboration with our results, Firestone et al. (2012) have demonstrated a significant increase in sperm motility in oligoasthenozoospermic samples exposed to a 30 -second infrared pulse of $50 \mathrm{~mW} / \mathrm{cm}$ at $905 \mathrm{~nm}$. As mentioned earlier, it is this wavelength that triggers an increase in light-sensitive Cytochrome $\mathrm{C}$ oxidase, a vital part of the energy generating machinery in the 
mitochondrial electron transport chain. These researchers also report that no significant increase in DNA damage occurred as compared with control samples, a finding reflected by our results as well. As mentioned from our experiments, low-level $900 \mathrm{~nm}$ IR exposure appears to have a positive, short-term effect on the motility of human spermatozoa. Thus, the intensity and duration of exposure in the nearinfrared region have a significant bearing on the cell response.

These current reports emphatically point to the stimulatory action of low level, short duration near-infrared radiation exposure to cells in-vitro, validating our premise that human sperm cells could be hyperactivated in-vitro on exposure to low dose near-IR. We believe that this could hold promise in enhancing in-vitro capacitation and fertilization technology.

\section{CONCLUSIONS}

The widespread, unrestrained use of nearinfrared radiation (NIR) in varied applications leads to increasing exposure of human life to this form of radiation and hence it is now the urgent need of the hour to verify the exact nature and implications of exposure to NIR. Investigations on the cellular injury following long term, high intensity exposures have revealed that generation of free radicals results in oxidative damage to cells and tissues, in addition to a direct impact. In recent years conversely, the biostimulatory effects of low level, near-infrared lasers have been elucidated. Research from our laboratory also confirms a beneficial effect of very low intensity, short duration near-infrared radiation. Paradoxically our earlier animal experimentation had indicated that near infrared radiation exposure for moderate durations resulted in a deleterious effect on mouse reproductive tissues. Hence, there is an evident intensity-duration factor that influences the impact of this most intriguing form of infrared radiation.

\section{REFERENCES}

[1] Aly Eman Mohamed and Mohamed Eman Saad.( 201). Effect of infrared radiation on the lens. Indian J Ophthalmol.: 59(2): 97-101.

[2] Ban Frangez H, Frangez I, Verdenik I, Jansa V, Virant KI. (2015). Photobiomodulation with light-emitting diodes improves sperm motility in men with asthenozoospermia. Lasers Med Sci 30:235-24

[3] Carlsen E, Giiwercman A, Keiding N, Skakkeback NE. (1992). Evidence for decreasing quality of semen during the past fifty years. British Medical Journal, 305:600613.

[4] Cheng CY and Mruk DD. (2012). The BloodTestis Barrier and Its Implications for Male Contraception. Pharmacol Rev. 64(1): 16-64.

[5] Cho Soyun, Mi Hee Shin, Yeon Kyung Kim , Jo-Eun Seo, Young Mee Lee , Chi-Hyun Park and Jin Ho Chung. (2009). Effects of Infrared Radiation and Heat on Human Skin Aging in vivo. Journal of Investigative Dermatology Symposium Proceedings. 14: 15-19.

[6] Eells JT, Gopalakrishnan S, Valter K. (2015). Near-Infrared Photobiomodulation in Retinal Injury and Disease. $d v$ Exp Med Biol. :854:437-441.

[7] Firestone RS, Esfandiari N, Moskovtsev SI, Burstein E, Videna GT, Librach C, Bentov Y, Casper RFJ. (2012). The effects of low-level laser light exposure on sperm motion characteristics and DNA damage. J. Androl. 33(3):469-73.

[8] Giacci MK, Hart NS, Hartz RV, Harvey AR, Hodgetts SI, Fitzgerald M. (2015). Method for the assessment of effects of a range of wavelengths and intensities of red/nearinfrared light therapy on oxidative stress in vitro. J Vis Exp.: 21: 86- 97.

[9] Gonzalez VC, Beheregaray AC, Peres BM, Sallis ES, Varela Junior AS, Trindade GS. (2015) Histopathological Analysis of UVB and IR Interaction in Rat Skin. Photochem Photobiol. 91(4):895-900.

[10] Highland HN and George LB. (2005). Effect of Infrared radiation on the reproductive function of adult male albino rats. Indian J. Environ. And Toxicol. 5 (1): 7-11.

[11] Highland HN, Bhatt SN and George LB. (2003). Biochemical and histoarchitectural alteration in mouse testis on exposure to infrared radiation. Toxicol Int. 10(1) : 23-29.

[12] Holick MF. (2016). Biological Effects of Sunlight, Ultraviolet Radiation, Visible Light, Infrared Radiation and Vitamin D for Health. Anticancer Res. : 36(3):1345-56.

[13] Hudson RD. Jr. (1969). Infrared System Engineering, John Wiley \& Sons. N.Y. 85 pp.

[14] Konig K, Tadir Y, Patriziol P, Berns WS and Tromberg BJ. (1966). Effect of ultraviolet exposure and near infrared lazer tweezers on human spermatozoa. Hum. Reprod., 11(10): 2162-2164

[15] Lenz P. (1995). In vivo excitation of photosensitizers by infrared light. Photochem. Photobiol., 62(2), 333-328.

[16] Li X, Liu J, Liang S, Sun C. (2014). 980$\mathrm{nm}$ infrared laser modulation of sodium channel kinetics in a neuron cell linearly mediated by photothermal effect. J Biomed Opt.:19(10):105002. 
[17] Linz Buoy George. (1990). A Study on the Effect of Infrared Radiation on the Human Crystalline Lens. Ph.D. thesis. Gujarat University, Ahmedabad.

[18] Lubart R, Friedmann H, Sinyakov M, Cohen N, Breitbart H. (1997). Changes in calcium transport in mammalian sperm mitochondria and plasma membranes caused by $780 \mathrm{~nm}$ irradiation. Lasers Surg Med.: 21(5):493-499.

[19] Mitsunaga M, Nakajima T, Sano K, KramerMarek G, Choyke PL, Kobayashi H. (2012). Immediate in vivo target-specific cancer cell death after near infrared photoimmunotherapy. BMC Cancer. 12:345.

[20] Overstreet JW, Samuels SJ, Day P, Hendrickx AG, Prahalada S, Mast T, Katz DF, Sakai C. (1988). Early indicators of male reproductive toxicity. Risk Anal. 8(1):21-6.

[21] Robert C, Bonnet M, Marques S, Numa M, and Doucet O. (2015). Low to moderate doses of infrared A irradiation impair extracellular matrix homeostasis of the skin and contribute to skin photodamage. Skin Pharmacol Physiol. 28(4):196-204.

[22] Roser M. (2016). Fertility Online. Ourworldindata.org. https://ourworldindata.org/fertility/ [Online Resource]

[23] Sakkas D, Alvarez JG. (2010). Sperm DNA fragmentation: mechanisms of origin, impact on reproductive outcome, and analysis. Fertil Steril 93: 1027-1036.
[24] Tanaka Y, Matsuo K. and Yuzuriha S. (2011). Near-Infrared Irradiation Non-thermally Affects Subcutaneous Adipocytes and Bones. Eplasty. 9:11-12.

[25] Tanaka Y, Tatewaki N, Nishida H, Eitsuka T, Ikekawa N, Nakayama J. 2012. Non-thermal DNA damage of cancer cells using nearinfrared irradiation. Cancer Sci. ; 103(8):146773.

[26] Tang J, Du Y, Lee CA, Talahalli R, Eells JT, Kern TS. (2013). Low-intensity far-red light inhibits early lesions that contribute to diabetic retinopathy: in vivo and in vitro. Invest Ophthalmol Vis Sci. 54(5):3681-3690.

[27] Vatansever F, and Hamblin MR. (2012). Far infrared radiation (FIR): its biological effects and medical applications.Photonics Lasers Med. 1(4) :255-266.

[28] Xia N, Peng F, Wang X, Zheng XL, Wan XP, Yuan W, Hou WS. (2014). Shortwavelength near infrared stimulation of the inner ear hair cells. Conf Proc IEEE Eng Med Biol Soc.2014:2633-2636.

[29] Yazdi SR, Bakhshi S, Jannat Alipoor F, Akhoond MR, Borhani S, Farrahi F, Lotfi Panah M, Sadighi Gilani MA. (2013). Effect of 830-nm diode laser irradiation on human sperm motility. Fertil Steril 4(Suppl 1):31-32

[30] Zeret MM, Synder WZ and Birenbaum L. (1976). Cataract after exposure to non-ionizing radiant energy. British J., Ophthalmology, 60, 632.

Citation: Hyacinth H, Linz-Buoy G. Near-Infrared Radiaton: An All Pervading Yet Lesser Known Environmental Stressor. International Journal of Research Studies in Medical and Health Sciences. 2017;2(3):13-20.

Copyright: (C) 2017 Hyacinth H, Linz-Buoy G. This is an open-access article distributed under the terms of the Creative Commons Attribution License, which permits unrestricted use, distribution, and reproduction in any medium, provided the original author and source are credited. 